\title{
Preparation of large aperture Photo-Detectors for the Hyper-Kamiokande
}

\section{Takuya Tashiro* for the Hyper-Kamiokande Collaboration}

Research Center for Cosmic Neutrino (RCCN), Institute for Cosmic Ray Research, University of Tokyo

E-mail: ttashiro@icrr.u-tokyo.ac.jp

Hyper-Kamiokande is a large water Cherenkov detector in Japan to explore various neutrino physics and discover a nucleon decay. Its construction started in 2020 and the mass production of a large aperture photo-detector is planned from the end of 2020. We developed a photomultiplier tube (PMT) with a $50 \mathrm{~cm}$ diameter, R12860 by Hamamatsu Photonics K.K. and installed over a hundred of them into Super-Kamiokande. A hundred PMTs were evaluated in Super-Kamiokande and compared with an R3600 PMT for Super-Kamiokande. It was confirmed that the resolutions and detection efficiency are doubly improved. A noise reduction of the PMT was also achieved with suppressing radioactive contamination in the glass. In addition, another large aperture photodetector using a micro channel plate was developed. A part of the detection wall with the optical acrylic window and several designs of a shockwave prevention cover are constructed for a test to demonstrate a coming construction work. A PMT calibration facility is also under preparation. We present the recent development and improved performance of the large-aperture photo-detectors.

40th International Conference on High Energy physics - ICHEP2020

July 28 - August 6, 2020

Prague, Czech Republic (virtual meeting) 


\section{Introduction}

Hyper-Kamiokande(HK) is the next generation water Cherenkov detector designed to explore wide range of physics targets including neutrino oscillation, neutrino astronomy, nucleon decay, and so on. HK is a cylindrical-shape detector filled with ultra-pure water of $260 \mathrm{kton}$, among which approximately 188 kton will be fiducial mass. The detector is separated into two parts. One is inner-detector(ID), which is the main active volume, and the other is outer-detector(OD), which covers ID to act as a veto against incoming particles. In HK, charged particles passing through the ultra pure water emit Cherenkov light, which is then detected by photodetectors mounted on the support frame. The photodetecors used in HK ID are large aperture PMTs with the diameter of 50 $\mathrm{cm}$, which have been developed for HK to enhance the overall experimental performance.

Throughout this document, the candidates of the ID PMTs, their performance, and preparation of their installation system are summarized.

\section{Candidates of large-aperture PMTs}

There are two types of candidates to be used in HK ID; One is Box\&Line PMT (shown in Fig.1), and the other is MCP PMT (shown in Fig.2). The evaluation of the performance of each $\mathrm{PMT}$ is described in this section.

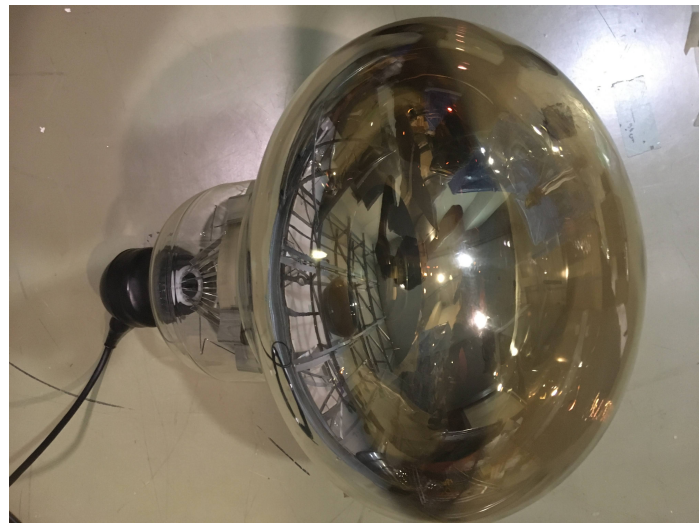

Figure 1: Box\&Line PMT

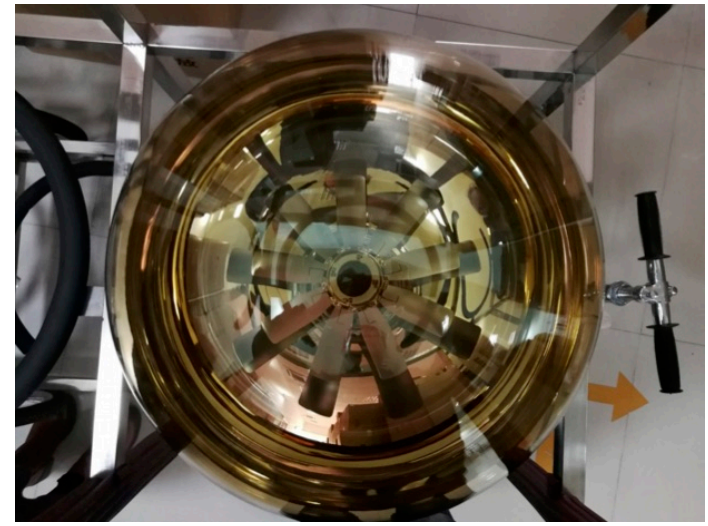

Figure 2: MCP PMT

\subsection{Box\&Line PMTs}

Box\&Line PMT is a product developed for HK by Hamamatsu photonics. It adopts a box-andline dynode arrangement as shown in Fig. 3 to achieve high detection efficiency, timing resolution, and charge resolution.

In 2018, 136 Box\&Line PMTs were installed into Super-Kamiokande(SK) to evaluate the performance in water.

The detection efficiency was measured using the NiCf calibration system[2]. In this system, a ball containing $\mathrm{Ni}$ and $\mathrm{Cf}$ is inserted into the $\mathrm{SK}$ tank and ${ }^{58} \mathrm{Ni}$ captures neutrons provided by ${ }^{252} \mathrm{Cf}$ to emit gamma rays with the energy of approximately $9 \mathrm{MeV}$. The number of photons detected 


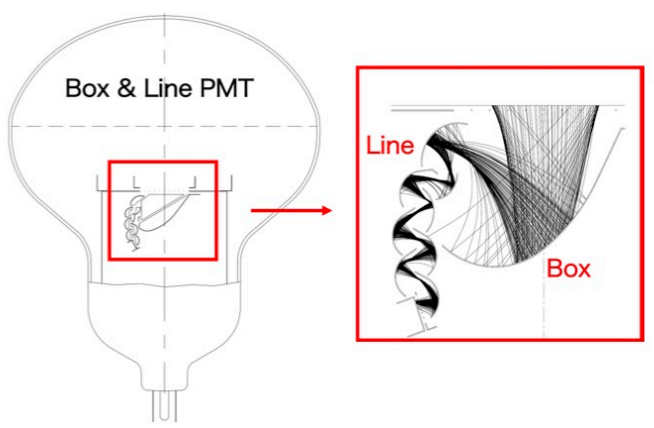

Figure 3: The dynode arrangement of Box\&Line PMTs.

by Box\&Line PMTs was compared to SK PMTs and the detection efficiency of Box\&Line PMTs was estimated to be $\sim 1.97$ times larger than SK PMTs in average. The distribution of the relative detection efficiency is shown in Fig.4

The charge and timing resolution were measured using the calibration system of the laser with the wavelength of $404 \mathrm{~nm}$ and $398 \mathrm{~nm}$, respectively. In this system, the photons were diffused by a diffuser ball inserted into the tank and then detected by the PMTs. As a result of the charge measurement, the relative charge resolution $\frac{\sigma}{\text { mean }}$ was estimated to be $\sim 27 \%$ and $\sim 54 \%$ for Box\&Line PMTs and SK PMTs, respectively. In the timing resolution measurement, the transittime-spread(T.T.S.) was estimated to be $\sim 1.50 \mathrm{~ns}$ and $\sim 2.96 \mathrm{~ns}$ for Box\&Line PMTs and SK PMTs, respectively. Hence, in the charge and timing resolution, approximately twice better performance was achieved in Box\&Line PMTs compared to SK PMTs. The distribution of the charge and timing resolution are shown in Fig.5 and Fig.6, respectively.

The rate of the dark noise is also one of the important property of PMTs. In HK PMTs, the rate is required to be as low as $4 \mathrm{kHz}$. The past investigation revealed that significant amount of dark noise is triggered by continuous pulses, the pulses that appear continuously within short time of $O(10) \mu \mathrm{s}$. One of the sources generating the continuous pulses is scintillation light triggered by decay of radioisotopes contained in the PMT bulbs. In Box\&Line PMT development, the raw materials of the PMT bulbs have been improved to reduce the contamination of radioisotopes. The amount of radioisotope contamination in SK PMT bulbs and Box\&Line PMT bulbs before and after the improvement are summarized in Table.1. The improvement of the raw materials succeeded in reducing the contamination to $\sim 1 / 2$.

The rate of dark noise was measured in the Box\&Line PMTs before and after the bulb improvement and it was probed that the reduction of radioisotope contamination resulted in lower rate of dark noise; The average rate after the improvement was $4.0 \mathrm{kHz}$, while it was originally $6.6 \mathrm{kHz}$ before the improvement.

\subsection{MCP PMTs}

MCP PMTs were developed for JUNO experiment[1] by North Night Vision Technology Co., Ltd. (NNVT) and now improved versions are prepared to meet the HK requirements. The performance of the HK MCP PMTs was measured using the setup built in Kamioka-mine. As results of the measurement, the charge resolution and the timing resolution were estimated to be 


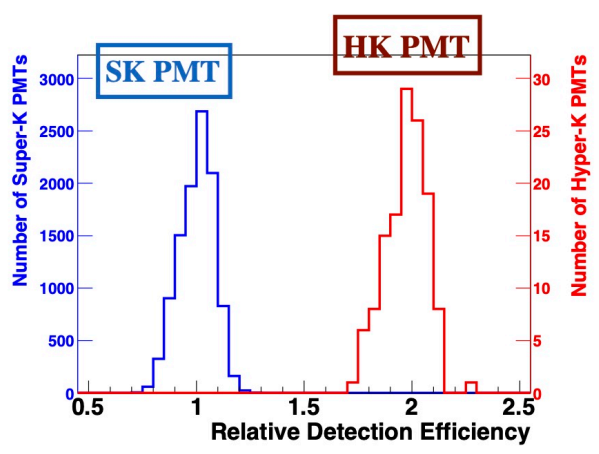

Figure 4: The detection efficiency distribution of SK PMTs and Box\&Line PMTs relative to the average of the SK PMTs.

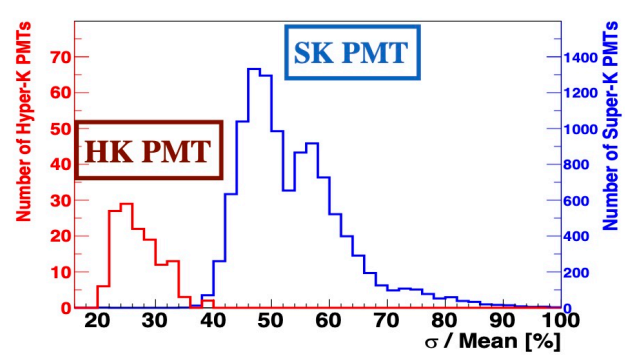

Figure 5: The charge resolution distribution of SK PMTs and Box\&Line PMTs.

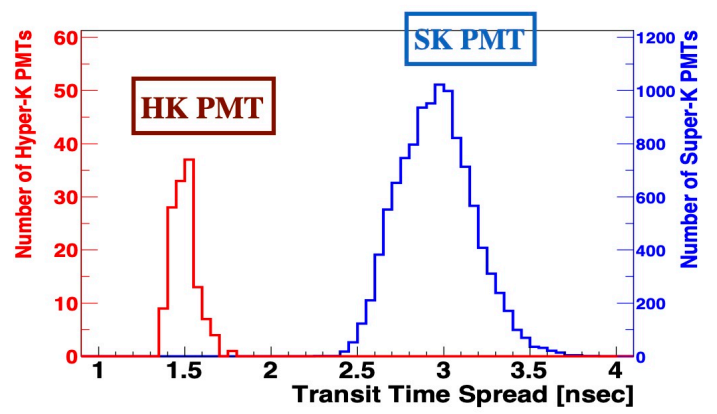

Figure 6: The timing resolution distribution of SK PMTs and Box\&Line PMTs.

\begin{tabular}{r|c|c|c} 
& SK PMTs & Box\&Line PMTs (Before improvement) & Box\&Line PMTs (After improvement) $]$ \\
\hline $\mathrm{U}[\mathrm{Bq} / \mathrm{kg}]$ & 5.5 & 5.4 & 2.9 \\
$\mathrm{Th}[\mathrm{Bq} / \mathrm{kg}]$ & 1.8 & 1.8 & 0.95 \\
${ }^{40} \mathrm{~K}[\mathrm{~Bq} / \mathrm{kg}]$ & 18.2 & 1.6 & 2.0 \\
\hline
\end{tabular}

Table 1: Table of the radioisotope contamination in PMT bulbs.

$\sim 40.3 \%$ and $1.8 \mathrm{~ns}$, respectively. These are better than the SK PMTs, whose charge and timing resolution are $54 \%$ and $3.0 \mathrm{~ns}$, respectively. The charge and timing distribution and their fit results are shown in Fig.7 and 8.

Since the HK tank is deeper than JUNO, higher pressure tolerance is required in HK MCP PMTs than the original design. The design was improved for HK and the water-pressure tolerance measurement proved that the pressure of $1.2 \mathrm{MPa}$, corresponding to the water depth of $120 \mathrm{~m}$, does not damage the MCP PMT. 


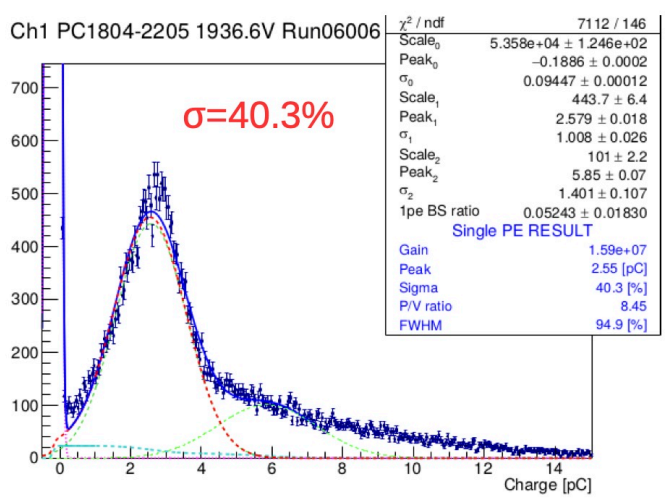

Figure 7: The charge distribution of MCP PMTs and its fit results.

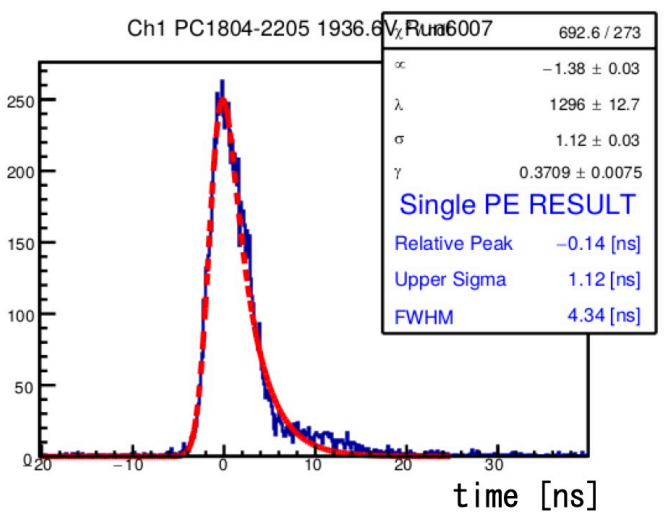

Figure 8: The timing distribution of MCP PMTs and its fit results.

\section{Covers and support structure}

In HK, PMTs will be stored in covers to avoid chain implosion. One of the considered design is Spanish cover (shown in Fig.9). As the preparation of Spanish cover, the basic design and pressure tolerance test were completed. The implosion test to prove the torrance against the implosion is scheduled in 2021. Another candidate design is Japanese cover (shown in Fig.10). In the preparation of Japanese cover, the basic design, pressure tolerance test, and the implosion test were already completed and it is proved to work under the HK configuration.

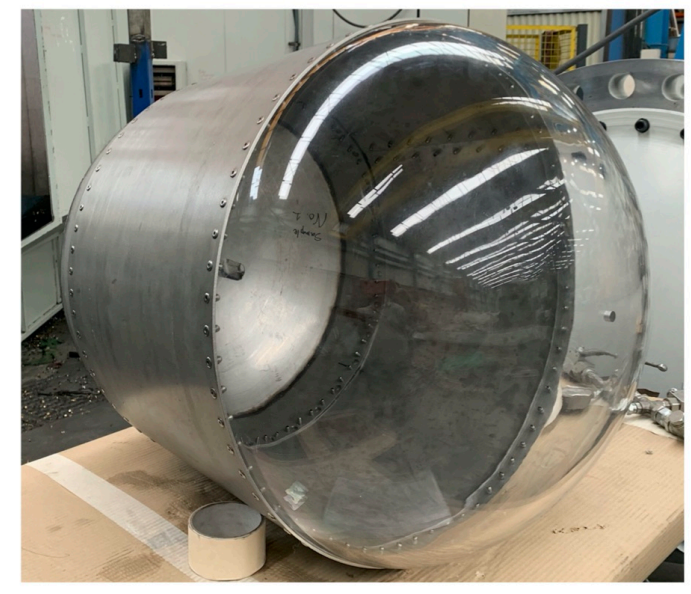

Figure 9: Spanish cover.

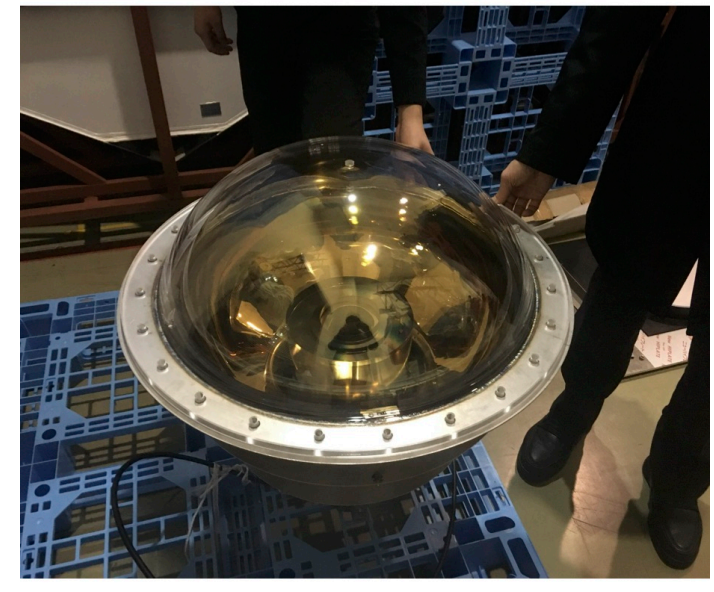

Figure 10: Japanese cover containing a Box\&Line PMT.

Design of PMT support structure and the installation system are ongoing. An actual-size mockup of the support structure was built in 2020 to perform installation test and to realize efficient and precise installation method. 


\section{Summary}

Preparation of large aperture PMTs for HK ID is ongoing. Two candidates, Box\&Line PMTs and MCP PMTs, have been developed and their performance was evaluated. In Box\&Line PMTs, the detection efficiency, charge resolution, and timing resolution are about twice better than SK PMTs and the dark noise rate is as low as $4 \mathrm{kHz}$. In MCP PMTs, both the charge and timing resolution are better than SK PMTs and enough pressure tolerance is achieved.

The covers and support structure are also prepared. In both Spanish and Japanese cover, the basic design and pressure tolerance test were completed. Implosion test for Spanish cover is scheduled in 2021. Designs of installation methods are being tested using the actual-size mockup frame to realize efficient and precise installation.

\section{References}

[1] Z. Djurcic et al. [JUNO], [arXiv:1508.07166 [physics.ins-det]].

[2] K. Abe, Y. Hayato, T. Iida, K. Iyogi, J. Kameda, Y. Kishimoto, Y. Koshio, L. Marti, M. Miura and S. Moriyama, et al. Nucl. Instrum. Meth. A 737 (2014), 253-272 doi:10.1016/j.nima.2013.11.081 [arXiv:1307.0162 [physics.ins-det]]. 